\title{
In Vivo Effectiveness of Orlistat in the Suppression of Human Colorectal Cancer Cell Proliferation
}

\author{
ALEKSANDRA CZUMAJ ${ }^{1}$, JUDYTA ZABIELSKA ${ }^{2}$, ALICJA PAKIET $^{3}$, ADRIANA MIKA $^{3}$, OLGA ROSTKOWSKA $^{4}$, \\ WOJCIECH MAKAREWICZ ${ }^{5}$, JAROSŁAW KOBIELA ${ }^{4}$, TOMASZ SLEDZINSKI ${ }^{1}$ and EWA STELMANSKA ${ }^{2}$ \\ ${ }^{1}$ Department of Pharmaceutical Biochemistry, Faculty of Pharmacy, \\ Medical University of Gdansk, Gdansk, Poland; \\ ${ }^{2}$ Department of Biochemistry, Faculty of Medicine, Medical University of Gdansk, Gdansk, Poland; \\ ${ }^{3}$ Department of Environmental Analysis, Faculty of Chemistry, University of Gdansk, Gdansk, Poland; \\ ${ }^{4}$ Department of General, Endocrine and Transplant Surgery, \\ Faculty of Medicine, Medical University of Gdansk, Gdansk, Poland; \\ ${ }^{5}$ Department and Clinic of Surgical Oncology, Faculty of Medicine, Medical University of Gdansk, Gdansk, Poland
}

\begin{abstract}
Background/Aim: Fatty acid synthase (FASN) provides palmitate for cell membrane formation in colorectal cancer $(C R C)$ cells, however, palmitate is also available in the blood of CRC patients. The aim of this study was to examine whether orlistat, a FASN inhibitor, is able to attenuate CRC cell growth despite the availability of extracellular palmitate. Materials and Methods: Palmitate concentrations were measured in serum from CRC patients and healthy controls. HT-29 CRC cells were treated with orlistat and palmitate. Results: Treatment of CRC cells with orlistat caused a dose-dependent inhibition of cell proliferation. In turn, delivery of extracellular palmitate at doses lower than those found in the serum of CRC patients reversed inhibition by orlistat concentrations of up to 10 $\mu M$. Conclusion: Inhibition of CRC cell proliferation by orlistat is reversed by palmitate which is present at high levels in the serum. Therefore, orlistat may be effective in vivo only at high concentrations.
\end{abstract}

Colorectal cancer (CRC) is one of the leading causes of mortality among cancer patients, and the search for new therapies is still underway. Due to advances in screening techniques and improvements in treatments, the death rate from colorectal cancer has been decreasing in the United States and Europe. However, a trend of increasing incidence of the disease has been observed in recently developed

Correspondence to: Tomasz Sledzinski, Department of Pharmaceutical Biochemistry, Faculty of Pharmacy, Medical University of Gdansk, Gdansk, Poland. Tel: +48 583491465, e-mail: tomasz.sledzinski@gumed.edu.pl

Key Words: Colorectal cancer, fatty acid synthase, orlistat, palmitate. countries (1). This cancer tends to affect men and women equally, but men tend to develop it at a younger age (2). CRC can often be sporadic; however, hereditary forms are also common in this entity (2). Recent evidence shows that $\mathrm{CRC}$ is associated with considerable changes in lipid composition and metabolism in cancer cells $(3,4)$. One of the alterations characteristic of many cancers is enhanced lipogenesis, a process that provides lipids for cell membrane formation in rapidly proliferating cancer cells (5). Some authors have reported overexpression of fatty acid synthase (FASN), the major enzyme which produces palmitic acid, in CRC tissue (6-8). Furthermore, FASN levels in the serum of colorectal cancer patients are significantly higher than that in healthy individuals; thus, this enzyme may be a potential tumor marker (9). FASN knockdown has been associated with the inhibition of CRC cell proliferation (10). Moreover, FASN expression increases with tumor progression in certain tumor types, including colorectal cancer and is associated with increased disease aggressiveness and diminished survival and response to classical chemotherapeutic agents (11-13). Thus, research on inhibitors of FASN as potential anticancer therapies is underway (5). Orlistat (tetrahydrolipstatin) is a drug used for the treatment of obesity. Orlistat is an inhibitor of pancreatic and gastric lipase, and its action leads to decreased absorption of dietary fat (14). This agent, which is administered by the oral route, is minimally absorbed by the gastrointestinal tract and reduces the lipid supply from outside sources. However, orlistat is also an inhibitor of FASN $(15,16)$. Three studies have reported the antiproliferative effect of orlistat on CRC cells (17-19). Generally, cancer cells show a preference for endogenous fatty acids; however, the delivery of exogenous fatty acids is also required to support some malignancies (20). Despite the inhibition of FASN by orlistat, it is possible 
that CRC cells take up palmitic acid from the bloodstream in quantities allowing further proliferation. Thus, the aim of this study was to examine whether treatment of CRC cells with palmitate can reverse the antiproliferative action of orlistat.

\section{Materials and Methods}

Patients. The study included serum and tissue samples from 66 patients (42 men and 24 women) with a mean age of $68.1 \pm 11.2$ years and a mean BMI of $26.1 \pm 5.3 \mathrm{~kg} / \mathrm{m}^{2}$. Patients presented with locally advanced cancer at stage $I(n=2)$ or stage II $(n=58)$ and 6 with regionally advanced malignancies with metastases to regional lymph nodes (stage III). None of the patients received preoperative neoadjuvant treatment. Tissue samples were collected from both the tumor and normal large intestinal mucosa at least $5 \mathrm{~cm}$ from the tumor interface. Each sample was divided into two parts: one was used for molecular analysis and the other was used for the preparation of routine hematoxylin and eosin (H\&E)-stained microscopy slides for histopathological examination. The material for the molecular studies was frozen in liquid nitrogen immediately after collection and stored in aliquots at $-80^{\circ} \mathrm{C}$ until analysis. Moreover, $5 \mathrm{ml}$ blood samples were collected from all CRC patients and from 20 healthy controls (10 men and 10 women; mean age, $44.0 \pm 13.1$ years; mean BMI $\left.25.6 \pm 4.4 \mathrm{~kg} / \mathrm{m}^{2}\right)$. The control group comprised healthy volunteers referred for an annual medical checkup who had demographic and socioeconomic characteristics similar to those of CRC patients. Fasting blood samples were collected into tubes without anticoagulant, left at room temperature for $30 \mathrm{~min}$ to allow clotting, and centrifuged at $3,000 \times g$ for $15 \mathrm{~min}$ and $4^{\circ} \mathrm{C}$. After centrifugation, serum samples were stored in aliquots at $-80^{\circ} \mathrm{C}$ until analysis. The protocol of the study was compliant with the Declaration of Helsinki of the World Medical Association and was granted approval from the Local Bioethics Committee at the Medical University of Gdansk (decision no. NKBN/487/2015). Written informed consent was obtained from all the subjects prior to the study.

Determination of FASN mRNA levels in patient tissues. Total RNA was purified from cancer and normal colon tissue from patients with CRC ( $n=44)$ using RNeasy Plus Universal Kits (Qiagen, Hilden, Germany). RNA was reverse transcribed using recombinant Moloney murine leukemia virus reverse transcriptase and random hexamer primers (RevertAid First Strand cDNA Synthesis Kit, Thermo Fisher Scientific, Waltham, MA, USA) according to the manufacturer's instructions. Duplicates of each sample were assayed via relative quantitative polymerase chain reaction (PCR) using a CFX Connect Real-Time System (Bio-Rad, Hercules, CA, USA). $\beta$-Actin was used as the reference gene. Melting analysis was carried out at the end of the amplification cycle to verify the nonspecific amplification. The cycle number at which any particular sample crossed that threshold (Ct) was then used to determine the fold difference. The fold

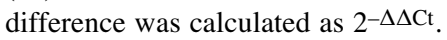

Solid phase extraction of lipids. Total lipids were extracted from serum aliquots with a chloroform:methanol mixture $(2: 1, \mathrm{v} / \mathrm{v})$ according to the procedure of Folch et al. (21). Solvents were evaporated under a nitrogen stream, and samples were re-dissolved in chloroform. Subsequently, lipids were fractionated using a solid phase extraction (SPE) protocol modified from that of Kaluzny et al. (22). Briefly, samples were loaded onto Strata NH2 aminopropyl cartridges $(3 \mathrm{ml}, 500 \mathrm{mg}, 55 \mu \mathrm{m})$ preconditioned with two volumes of hexane. Acylglycerides and cholesterol were washed out with 6 $\mathrm{mL}$ of chloroform:isopropanol $(2: 1, \mathrm{v} / \mathrm{v})$ and discarded. Free fatty acids (FFAs) were eluted with $2 \%$ acetic acid in diethyl ether and dried under a nitrogen stream.

Analysis of palmitate content by gas chromatography-mass spectrometry $(G C / M S)$. Fatty acid methyl esters (FAME) were prepared for analysis of palmitate content by GC/MS. FFA derivatization was performed using a mixture of $10 \%$ boron trifluoride in methanol at $55^{\circ} \mathrm{C}$ for $1.5 \mathrm{~h}$, and $1 \mathrm{ml}$ of water was added. FAME were extracted three times with $1 \mathrm{ml}$ hexane, and the solvent was evaporated under a nitrogen stream. Analysis was conducted with a GC-EI-MS QP-2010SE (Shimadzu, Kyoto, Japan). FAME were separated on a Zebron ZB-5MSi capillary column (30 m length $\times 0.25 \mathrm{~mm}$ i.d. $\times 0.25 \mu \mathrm{m}$ film thickness) with the following GC oven temperature program: $60-300^{\circ} \mathrm{C}\left(4^{\circ} \mathrm{C} / \mathrm{min}\right)$ with a $60-\mathrm{min}$ overall run time. Helium was used as the carrier gas, and the column head pressure was set at $100 \mathrm{kPa}$. The electron impact source for mass spectrometric detection was operated at $70 \mathrm{eV}$. Acquisition of mass spectra was conducted in full scan mode with a mass scan range of $\mathrm{m} / \mathrm{z}$ 45-700. 19-Methylarachidic acid was used as the internal standard. Identification of fatty acids was performed using reference standards (37 FAME Mix, Sigma-Aldrich, St Louis, MO, USA) and the NIST 2011 reference library.

Measurement of free fatty acid concentrations. As the GC/MS analysis provides a percent fatty acid profile, to calculate the exact concentration values, the total concentration of free fatty acids was measured by the enzymatic method. Concentration determination was based on an in vitro enzymatic colorimetric assay method by a HR Series NEFA-HR (2) (FUJIFILM Wako Chemicals Europe $\mathrm{GmbH}$, Neuss, Germany). Briefly, the standard curve was determined using a standard kit included in the assay. Seven microliters of each investigated serum sample were placed in a measuring dish, and two reagents were subsequently added in minute 1 (reagent 1 ) and minute 3 (reagent 2). The addition of reagent 1 , which contained acyl-CoA synthase, to the sample resulted in acyl-CoA formation, the acyl-CoA was further oxidized by acyl-CoA oxidase that resulted in $\mathrm{H}_{2} \mathrm{O}_{2}$ formation. Then, in the reaction catalyzed by peroxidase (reagent 2), $\mathrm{H}_{2} \mathrm{O}_{2}, 3$-Methyl-NEthyl-N-( $\beta$-Hydroxyethyl)-Aniline (MEHA) and 4-aminoantipyrine (4-AA) formed bluish-purple pigments. The NEFA concentration was obtained by measuring the absorbance of the bluish-purple pigment in maximum absorption mode at $550 \mathrm{~nm}$. Measurements for each sample were performed twice for verification of accuracy.

Cell culture. The human colon adenocarcinoma cell line (HT-29) was obtained from American Type Culture Collection (Manassas, VA, USA). HT-29 cells were cultured in $100 \mathrm{~mm}$ culture dishes in McCoy's 5A Modified Medium containing 10\% heat-inactivated fetal bovine serum at the atmosphere of $5 \% \mathrm{CO}_{2}, 95 \%$ air at $37^{\circ} \mathrm{C}$.

Treatment with orlistat. Orlistat was dissolved in dimethyl sulfoxide (DMSO), (Sigma-Aldrich). HT- 29 cells were seeded in 6 -well culture plates at $0.2 \times 10^{6}$ cells/well and at proper confluency cells were switched from the standard medium to either: 1) the same fresh medium 2) medium with DMSO 3) 
A

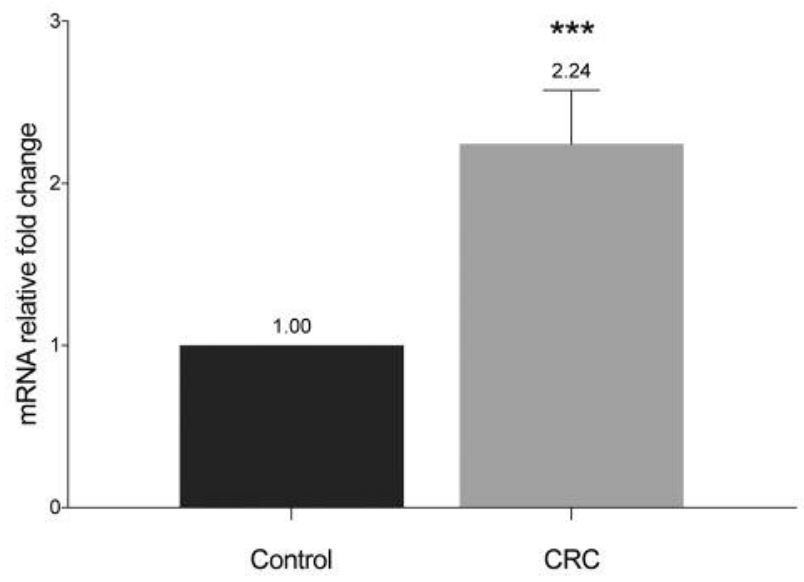

B

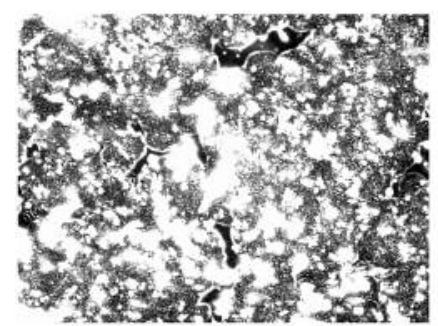

Control

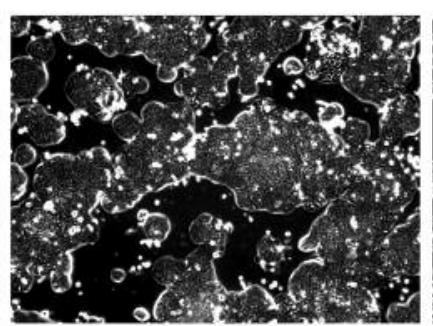

$6 \mu \mathrm{M}$ orl.

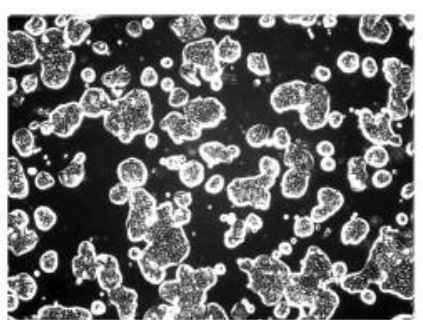

$10 \mu \mathrm{M}$ orl.

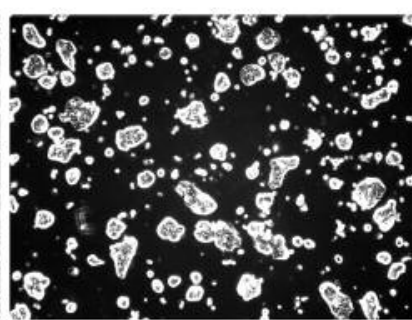

$50 \mathrm{MM}$ orl.

C

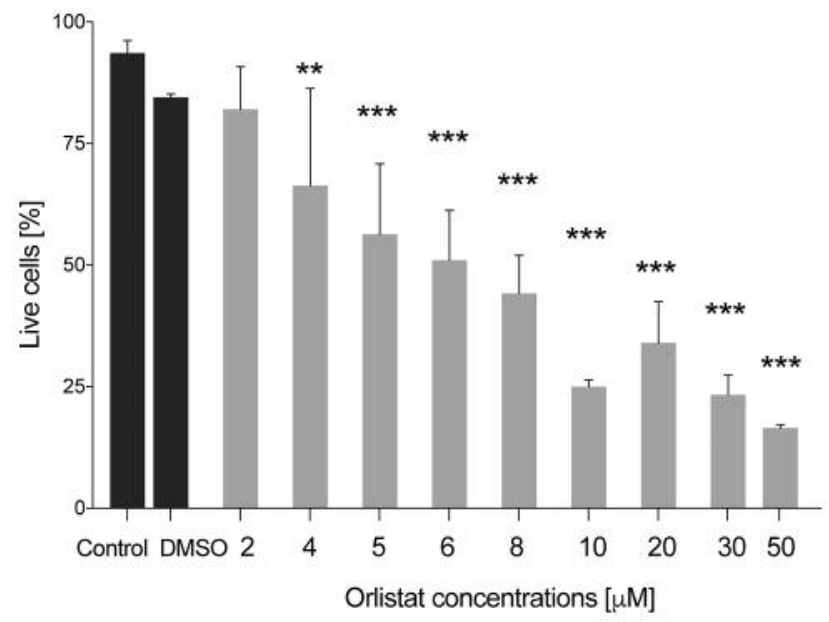

Figure 1. FASN mRNA levels in colon cancer tissue from CRC patients (A). Influence of increasing orlistat concentrations on HT-29 cell growth $(B)$, and number $(C)$ after culture for $72 \mathrm{~h}$. Cultured cells were imaged with an EVOS XL Cell Imaging System. Cell counts were determined on a Countess II Automated Cell Counter Cell. The results originate from at least three independent experiments. Control groups are cells cultured in medium; "DMSO" are cells cultured in medium containing DMSO. Data shown are the means \pm SEMs. *Significantly different from the control cell culture without orlistat at $p<0.05$.

medium supplemented with different concentrations of orlistat 4) medium supplemented with orlistat and palmitic acid. The cells were incubated for $72 \mathrm{~h}$.

Cell imaging and counting. Cultured cells were photographed with EVOS XL Cell Imaging System (ThermoFisher Scientific). After that cells were trypsinized and counted on Countess II Automated Cell Counter (Life Technologies, Carlsbad, CA, USA).

Statistical analysis. Statistical analyses were performed in GraphPad Prism 8. Results are presented as mean \pm SEM of at least three independent experiments. Statistical significance of in vitro 


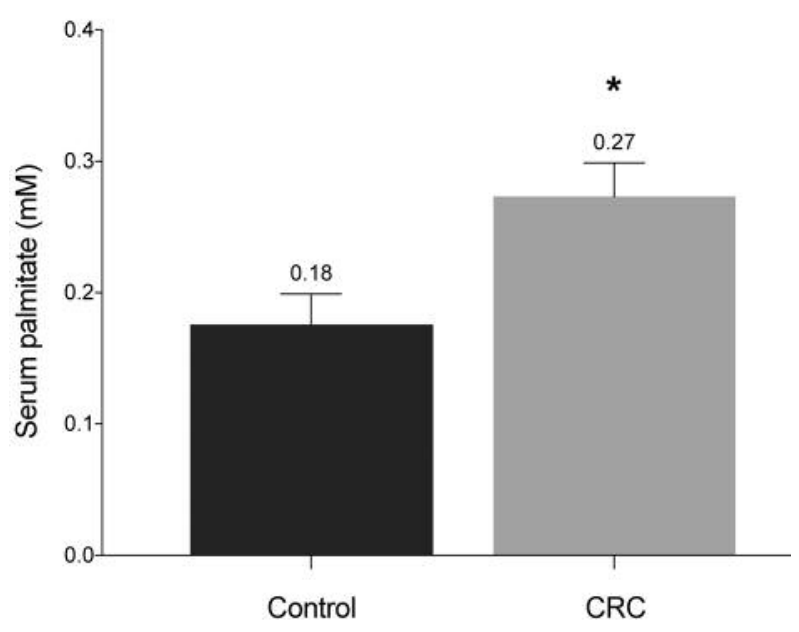

Figure 2. Concentration of palmitate in the serum of colorectal cancer patients. Data are presented as the means \pm SEMs. *Significantly different from control heathy individuals at $p<0.05$.

experiments was examined by one-way analysis of variance (ANOVA) with Dunnett and Tukey's HSD post-hoc. For gene expression and FFA assessment, two-tailed Student $t$-test was used. $p<0.05$ was considered statistically significant.

\section{Results}

The mRNA levels of FASN in colon cancer tissue from CRC patients were significantly higher than those in normal intestinal mucosa (Figure 1A). Treatment of CRC HT-29 cells with increasing concentrations of orlistat led to progressive inhibition of HT-29 cell growth (Figure 1B) and a decrease in the cell number (Figure 1C). A 50\% inhibition of HT-29 cell proliferation occurred after treatment with 6-8 $\mu \mathrm{M}$ orlistat. Solid phase extraction followed by GC/MS revealed that the serum palmitate content accounted for nearly $40 \%$ of the FFA fraction corresponding to a concentration of approximately $300 \mu \mathrm{M}$, which was significantly higher than that in healthy subjects (Figure 2). Thus, CRC cells were incubated with $300 \mu \mathrm{M}$ palmitate to examine whether the concentration of exogenous palmitate found in the circulation of CRC patients can reverse the antiproliferative action of orlistat. However, under in vitro conditions, palmitate at concentrations greater than $100 \mu \mathrm{M}$ inhibited CRC HT-29 cell proliferation (not shown). Thus, cells were treated with $50 \mu \mathrm{M}$ palmitate and increasing concentrations of orlistat. We found that in cell cultures treated with orlistat at concentrations up to $10 \mu \mathrm{M}$, cotreatment with $50 \mu \mathrm{M}$ palmitate almost completely restored cell viability (Figure $3 \mathrm{~A})$. The addition of $50 \mu \mathrm{M}$ palmitate also increased the viability of cells treated with higher concentrations of orlistat, but the viability percentages were significantly lower than those in control cells: approximately 50\% that of control cells in cultures treated with $50 \mu \mathrm{M}$ orlistat and $50 \mu \mathrm{M}$ palmitate (Figure 3B).

\section{Discussion}

Fatty acid synthase plays an important role in tumor cell development and survival because it provides palmitic acids needed for the synthesis of membrane phospholipids during rapid cell growth and proliferation. Moreover, endogenously synthesized fatty acids act as posttranslational protein modifiers and modulate gene expression (24). Palmitate and palmitate-derived lipids are known to play essential roles in modulating signal transduction and function of proteins associated with signaling pathways that regulate cell growth and survival (24). Furthermore, recent studies have suggested that endogenously synthesized fatty acids are utilized by $\beta$-oxidation in CRC cells, which supports cancer cell survival, particularly under conditions of energy stress (8). Several studies have shown that FASN is specifically upregulated in many types of malignancies, including pancreatic, prostate, breast, lung, bladder, ovarian and colon cancer $(15,19,25)$. Our study also documented FASN overexpression in tumor tissues from colorectal cancer patients (Figure 1A). Elevated levels of FASN in tumor cells have been linked to poor prognosis, disease aggressiveness, aggressive tumor phenotypes and increased risk of death (13). Because of the strong link between FASN expression and cancer, this enzyme has become an attractive target for therapeutic intervention. Several natural and synthetic FASN inhibitors (e.g., cerulenin, C75, epigallocatechin-3gallate, triclosan, and luteolin) have been shown to inhibit cancer cell growth $(13,26)$. Orlistat, an irreversible inhibitor of pancreatic and gastric lipases, has also been shown to selectively inhibit FASN activity (16). This agent acts as a tightly binding irreversible inhibitor of serine hydrolase activity within the thioesterase domain of FASN (26). It has also been shown that orlistat inhibits FASN activity and induces cell death in many tumor cell lines in vitro $(13,15-18,27,28)$ and that it is effective in inhibiting tumor growth in xenograft models in vivo (16, $17,28)$. Our study confirmed that orlistat effectively inhibits colorectal cancer cell proliferation, similar to the findings of previous studies (Figure 1B-C). This agent has also been shown to slightly prolong the survival of mice (fed a high-fat diet) with fatal hereditary gastrointestinal cancer (29). Moreover, the in vivo antitumor action of orlistat has been demonstrated in two models of murine melanoma $(30,31)$. However, it has been established that treatment of mice with orlistat was associated with a significant increase in the number of aberrant colonic crypt foci, which are putative precursors of colon cancer 
A

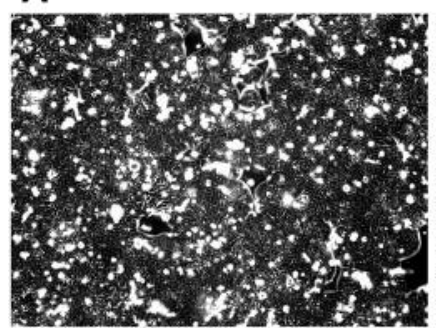

Control

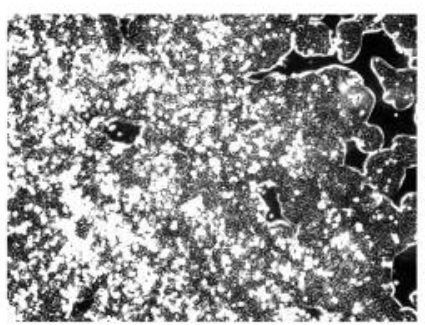

Control

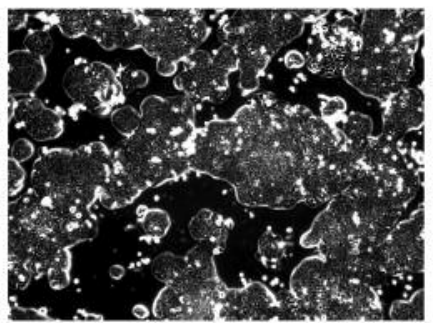

$6 \mu \mathrm{M}$ orl.

$\downarrow$

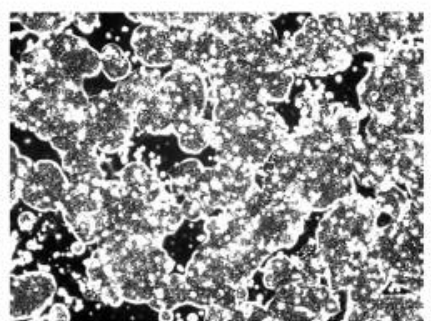

$6 \mu \mathrm{M}$ orl.

+50 $\mu \mathrm{M}$ palm.

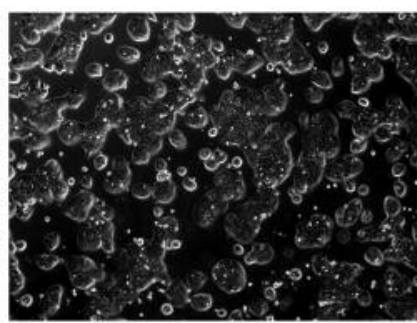

$10 \mu \mathrm{M}$ orl.

$\downarrow$

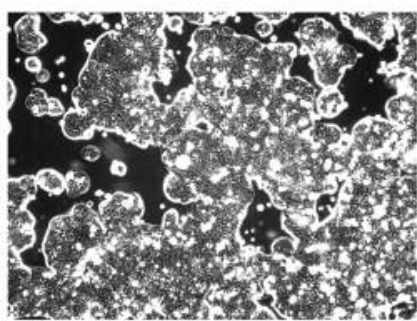

$10 \mu \mathrm{M}$ orl.

$+\mathbf{5 0} \mu \mathrm{M}$ palm.

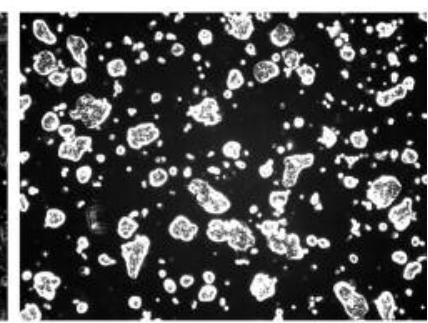

$50 \mu \mathrm{M}$ orl.

$\downarrow$

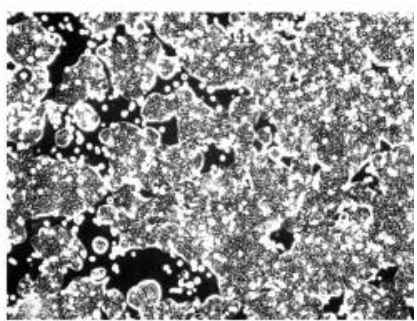

$50 \mathrm{uM}$ orl.

$+\mathbf{5 0} \mu \mathrm{M}$ palm.

B

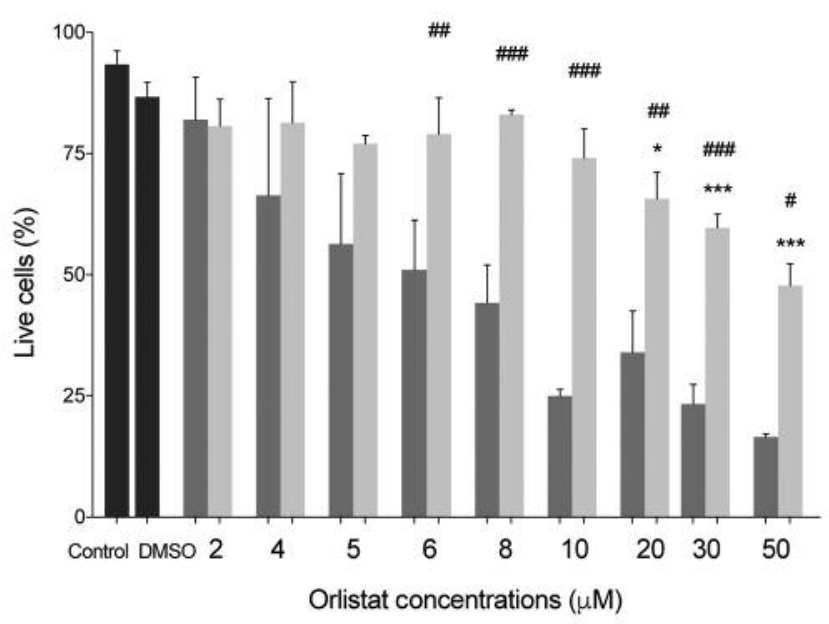

Figure 3. The effect of co-treatment with $50 \mu \mathrm{M}$ palmitate and increasing concentrations of orlistat on HT-29 cell growth (A) and number (B) after culture for $72 \mathrm{~h}$. Cultured cells were imaged with an EVOS XL Cell Imaging System. Cell counts were determined on a Countess II Automated Cell Counter. The results originate from at least three independent experiments. Control groups are cells cultured in medium; "DMSO" are cells cultured in medium containing DMSO. Brighter bars-cells were incubated with palmitate. Data are shown as the means \pm SEMs. *Significantly different from the control cell culture without orlistat at $p<0.05$; "Significantly different from cells cultured with the same dose of orlistat without palmitate at $p<0.05$.

(32). Recent studies have indicated that this drug could reduce local $O^{6}$-methylguanine methyltransferase mediated protection against DNA damage provoked by DNA-methylating compounds in epithelial cells of the gastrointestinal tract, thus favoring chemical carcinogenesis (33). However, no evidence of an increased risk of colorectal cancer in humans after the initiation of orlistat has been found (34).
The most important and original result of our study is the demonstration that the antiproliferative effect of orlistat is abolished by the addition of palmitate (a product of FASN activity). It should be noted that palmitate (whose production is inhibited by orlistat) is present in the circulation and is available to cells. The reversal of orlistat action after palmitate addition has also been observed in a pancreatic cancer cell line (PANC-1) (15) and the human hepatoma cell 
line Hep3B (27). Furthermore, we showed that the concentration of free palmitate in the blood of colorectal cancer patients is significantly higher than that in control subjects (Figure 2). We demonstrated for the first time that the addition of palmitate at an even lower concentration than that found in patients' blood almost completely reversed the effect of orlistat on cultured HT-29 cells (Figure 3). These results indicate that the potential application of orlistat in the pharmacotherapy of colorectal cancer may be ineffective unless high concentrations of this drug are used. It should be considered that the antiproliferative effects of this agent may be modulated by the blood concentration of palmitate in patients. It is worth mentioning that cancer cells generally show a preference for endogenously synthetized fatty acids, but the provision of exogenous fatty acids may also be required in some malignancies (3). In vivo studies have indicated that FASN plays a central role in tumor cell development and growth, even in the presence of high levels of circulating fatty acids. Knockdown or pharmacological inhibition of FASN selectively induces the death of cancer cells and a reduction in the tumor volume in xenograft mouse models, with only a minimal effect on normal cells $(16,17)$. This finding indicates that the clinical application of FASN inhibitors may be reasonable and that the mechanism of orlistat's action and effects may be different in vivo than in vitro.

It is worth noting that FASN is not the only target of orlistat in cells (35); thus, other possible targets of this agent should be considered. A chemical proteomic approach has identified seven new orlistat targets: heat shock protein 90 (HSP90AB1), $\beta$-tubulin, annexin A2, glyceraldehyde-3phosphate dehydrogenase (GAPDH) and three ribosomal proteins (PRL14, PRL9 and PRL7a) (36). Some of these targets were further validated by experimental techniques such as western blotting, recombinant protein expression and site-directed mutagenesis. These findings may have important implications for considering orlistat as a potential anticancer drug because several of these new targets play a role in tumor biology (35). Thus, multiple cellular pathways can be simultaneously involved in the action of orlistat as a multitarget drug. However, our study showing that palmitate abolishes the effect of orlistat indicates that FASN inhibition is the main mechanism by which orlistat acts in colorectal cancer cells. On the other hand, the possibility that a new orlistat target may explain the effectiveness of orlistat in inhibiting xenograft tumor growth in mouse models $(16,17)$ and the previously described in vitro effects beyond the inhibition of fatty acid synthesis cannot be excluded. These effects include the inhibition of protein synthesis (18), induction of ER stress-regulated gene expression (18), induction of apoptosis via caspase-3 activation (17), and inhibition of the cell cycle by arresting cells at the $G_{1}$ phase $(17,27)$. Of course, these processes are essentially related to
FASN activity. Several studies have revealed important connections between lipid synthesis pathways and ER stress response $(18,37)$, lipid metabolism, microtubule function (38), and various signaling pathways driving tumor cell proliferation (24). As described above, palmitate is used not only as a substrate for the synthesis of other lipids but also participates in posttranslational modifications of many proteins, affecting their activity. For example, tubulins are translationally modified by acylation with palmitate. FASN inhibition significantly reduces tubulin palmitoylation, disrupting microtubule organization in tumor cells (39). It is worth noting that $\beta$-tubulin is one of the newly identified orlistat targets, indicating that the inhibition of tumor cell growth by orlistat can occur through various interacting mechanisms. Another example concerns the palmitoylation of Wnt proteins which are secreted, palmitoylated glycoproteins important in embryonic development and human cancers. It has been shown that FASN inhibition disrupts oncogenic signaling and tumor growth in xenograft models through the inhibition of pathways that include Wnt/ $\beta$-catenin and c-Myc (24).

It is also worth mentioning another mechanism that - in our opinion - has not been considered to date. Cancer cells require increased concentrations of cholesterol (in addition to phospholipids) for rapid cell growth and proliferation. However, the concentration of cholesterol in the membranes must be strictly controlled, so excess cholesterol is stored in an esterified form in lipid droplets. Palmitate and palmitatederived fatty acids (mainly oleic acid) are substrates in the esterification of cholesterol catalyzed by acyl- coenzyme A: cholesterol acyltransferase (ACAT). Inhibition of this enzyme has been shown to increase the levels of free cholesterol in cell membranes and to inhibit the growth and division of tumor cells (40). It cannot be ruled out that inhibition of FASN leads to a reduction in cholesterol esterification and, consequently, an increase in the level of free cholesterol, which is toxic to tumor cells. Yekaterina et al. demonstrated that overexpression of FASN led to the accumulation of lipid droplets in colorectal cancer cells (8). Furthermore, Salah et al. recently showed that orlistat significantly decreased the total cholesterol concentration in tumor tissue from mice (28). We hypothesize that this mechanism can be the cause of further substrate deficiency in the esterification and storage of cholesterol. The examination of the relationship between cholesterol metabolism and FASN inhibition in CRC cells seems to be important and requires further investigation.

In conclusion, we demonstrated that treatment of CRC cells with orlistat caused a dose-dependent inhibition of cell proliferation. The most important result of our study is the prevention of orlistat-induced HT-29 cell death by the addition of exogenous palmitate at doses lower than those found in the serum of CRC patients. This result indicates that 
FASN inhibition is the main mechanism of action by which orlistat inhibits the proliferation of CRC cells. Our results indicated that the potential application of orlistat in the pharmacotherapy of colorectal cancer may be ineffective unless high concentrations of this drug are used.

\section{Conflicts of Interest}

The Authors declare no conflicts of interest. The funders had no role in the design of the study; in the collection, analyses, or interpretation of data; in the writing of the manuscript, or in the decision to publish the results.

\section{Authors' Contributions}

Acquisition of clinical data: O.R., W.M., J.K.; Experimental work: A.C., J.Z., A.P., AM; Data analysis: A.M., O.R, W.M., J.K.; Writing - original draft: A.C., J.Z., E.S.; Writing - review \& editing: T.S., A.M., W.M., J.K., E.S.; Supervision: T.S, E.S. All Authors gave final approval for publication.

\section{Funding}

This research was supported by the National Science Centre of Poland (grant no. 2016/22/E/NZ4/00665) and Medical University of Gdansk (grants no. ST-40, ST-41 and ST-89).

\section{References}

1 Butler LM, Yuan JM, Huang JY, Su J, Wang R, Koh WP and Ong ChN: Plasma fatty acid and risk of colon and rectal cancers in the Singapore Chinese Health Study. NPJ Precis Oncol 1(38): 1-9, 2017. PMID: 29872717. DOI:10.1038/s41698-017-0040-z

2 Soyano AE, Baldeo C and Kasi PM: Case report BRCA mutation and its association with colorectal cancer. Clin Colorectal Cancer 17(4): 647-650, 2018. PMID: 30033118. DOI: 10.1016/j.clcc.2018.06.006

3 Pakiet A, Stepnowski P, Sledzinski T and Mika A: Changes in lipids composition and metabolism in colorectal cancer: a review. Lipids Health Dis 18(29): 2019. PMID: 30684960. DOI: 10.1186/s12944-019-0977-8

4 Mika A, Kobiela J, Czumaj A, Chmielewski M, Stepnowski P and Sledzinski T: Hyper-elongation in colorectal cancer tissue cerotic acid is a potential novel serum metabolic marker of colorectal malignancies. Cell Physiol Biochem 41(2): 722-730, 2017. PMID: 28214830. DOI: 10.1159/00045843

5 Swierczynski J, Hebanowska A and Sledzinski T: Role of abnormal lipid metabolism in development, progression, diagnosis and therapy of pancreatic cancer. World $\mathbf{J}$ Gastroenterol 20(9): 2279-2303, 2014. PMID: 24605027. DOI: 10.3748/wjg.v20.i9.2279

6 Rashid A, Pizer ES, Moga, M, Milgraum LZ, Zahurak M, Pasternack GR, Kuhajda FP and Hamilton SR: Elevated expression of fatty acid synthase and fatty acid synthetic activity in colorectal neoplasia. Am J Pathol 150(1): 201-208, 1997. PMID: 9006336.

7 Ogino S, Nosho K, Meyerhardt JA, Kirkner GJ, Chan AT, Kawasaki T, Giovannucci EL, Loda M and Fuchs ChS: Cohort Study of Fatty Acid Synthase Expression and Patient Survival in
Colon Cancer. J Clin Oncol 26(35): 13-15, 2008. PMID: 18955444. DOI: $10.1200 / J C O .2008 .18 .2675$

8 Zaytseva YY, Harris JW, Mitovl MI, Kim JT, Butterfield DA, Lee EY, Weiss HL, Gaol T and Evers BM: Increased expression of fatty acid synthase provides a survival advantage to colorectal cancer cells via upregulation of cellular respiration. Oncotarget 6(22): 2015. PMID: 25970773. DOI: 10.18632/oncotarget.3783.

9 Long Q, Yi Y, Qiu J, Xu CH and Huang PL: Fatty acid synthase (FASN) levels in serum of colorectal cancer patients: correlation with clinical outcomes. Tumor Biol 35: 3855-3859, 2014. PMID: 24430360. DOI: $10.1007 / \mathrm{s} 13277-013-1510-8$

10 Wang H, Xi Q and Wu G: Fatty acid synthase regulates invasion and metastasis of colorectal cancer via Wnt signaling pathway. Cancer Medicine, 2016. PMID: 27139420. DOI: 10.1002/ cam4.711

11 Zaytseva YY, Elliott VA, Rychahou P, Mustain WC, Kim JT, Valentino J, Gao T, O'Connor KL, Neltner JM, Lee EY, Weiss HL and Mark EB: Cancer cell-associated fatty acid synthase activates endothelial cells and promotes angiogenesis in colorectal cancer. Carcinogenesis 35(6): 1341-1351, 2014. PMID: 24510238. DOI: $10.1093 /$ carcin/bgu042

12 Zaytseva YY, Rychahou PG, Gulhati P, Elliott VA, Mustain WC, O'Connor K, Morris AJ, Sunkara M, Weiss HL, Lee EY and Evers BM. Inhibition of fatty acid synthase attenuates CD44associated signaling and reduces metastasis in colorectal cancer. Cancer Res 72(6): 1504-1517, 2012. PMID: 22266115. DOI: 10.1158/0008-5472.CAN-11-4057

13 Sadowski MC, Pouwer RH, Gunter JH, Lubik AA, Quinn RJ and Nelson CC: The fatty acid synthase inhibitor triclosan: repurposing an anti-microbial agent for targeting prostate cancer. Oncotarget 5(19): 9362-9381, 2015. PMID: 25313139. DOI: 10.18632/oncotarget.2433

14 Yanovski SZ and Yanovski JA: Long-term drug treatment for obesity: A systematic and clinical review. JAMA 311(1): 74-86, 2014. PMID: 24231879. DOI: 10.1001/jama.2013.281361.Longterm

15 Sokolowska E, Presler M, Goyke E, Milczarek R, Swierczynski $\mathrm{J}$ and Sledzinski T: Orlistat reduces proliferation and enhances apoptosis in human pancreatic cancer cells (PANC-1). Anticancer Res 37(11): 6321-6327, 2017. PMID: 29061815. DOI: 10.21873 /anticanres.12083

16 Kridel SJ, Axelrod F, Rozenkrantz N and Smith JW: Orlistat is a novel inhibitor of fatty acid synthase with antitumor activity. Cancer Res 64: 2070-2075, 2004. PMID: 15026345.

17 Chuang HY, Chang YF and Hwang JJ: Antitumor effect of orlistat, a fatty acid synthase inhibitor, is via activation of caspase-3 on human colorectal carcinoma-bearing animal. Biomed Pharmacother 65(4): 286-292, 2011. PMID: 21723078. DOI: 10.1016/j.biopha.2011.02.016

18 Little JL, Wheeler FB, Fels DR, Koumenis C and Kridel SJ: Inhibition of fatty acid synthase induces endoplasmic reticulum stress in tumor cells. Cancer Res 67(3): 1262-1270, 2007. PMID: 17283163. DOI: 10.1158/0008-5472.CAN-06-1794

19 Cervantes-Madrid D and Dueñas-González A: Antitumor effects of a drug combination targeting glycolysis, glutaminolysis and de novo synthesis of fatty acids. Oncol Rep 34(11): 1533-1542, 2015. PMID: 26134042. DOI: 10.3892/or.2015.4077

20 Cao D, Song X, Che L, Li X, Pilo MG and Vidili G: Both de novo synthetized and exogenous fatty acids support the growth of hepatocellular carcinoma cells. Liver Int 37(1): 80-89, 2018. PMID: 27264722. DOI: 10.1111/liv.13183 
21 Folch J, Lees $M$ and Stanley GH: A simple method for the isolation and purification of total lipides from animal tissues. $\mathbf{J}$ Biol Chem 226(1): 497-509, 1957. PMID: 13428781.

22 Kaluzny MA, Duncan LA, Merritt MV and Epps DE: Rapid separation of lipid classes in high yield and purity using bonded phase columns. J Lipid Res 26(1): 135-140, 1985. PMID: 3973509.

23 Vian L, Vincent J, Maurin J, Fabre I, Giroux J and Cano JP: Comparison of three in vitro cytotoxicity assays for estimating surfactant ocular irritation. Toxic in Vitro 9(2): 185-190, 1995. PMID: 20650078. DOI: 10.1016/0887-2333(94)00200-e

24 Heuer TS: De novo palmitate synthesis supports oncogenic signalling and tumor growth through diverse mechanisms: implications for FASN-targeted therapeutics. J Cell Signal 1(3): 1-4, 2016. DOI: $10.4172 / 2576-1471.1000124$

25 Kuhajda FP: Fatty acid synthase and cancer: new application of an old pathway. Cancer Res 66(12): 5977-5981, 2006. PMID: 16778164. DOI: 10.1158/0008-5472.CAN-05-4673

26 Lupu R and Menendez JA: Pharmacological inhibitors of fatty acid synthase (FASN) -catalyzed endogenous fatty acid biogenesis: A new family of anti-cancer agents? Curr Pharm Biotechnol 7: 483-494, 2006. PMID: 17168665. DOI: 10.2174/ 138920106779116928

27 You BJ, Chen LY, Hsu PH, Sung PH, Hung YCh and Lee HZ: Orlistat displays antitumor activity and enhances the efficacy of paclitaxel in human hepatoma Hep3B cells. Chem Res Toxicol 32(2): 255-264, 2019. PMID: 30667213. DOI: 10.1021/ acs.chemrestox.8b00269

28 Saleh A, Elfayoumi HM, Youns M and Barakat W: Rutin and orlistat produce antitumor effects via antioxidant and apoptotic actions. Naunyn Schmiedebergs Arch Pharmacol 392: 165-175, 2019. PMID: 30465055. DOI: 10.1007/s00210-018-1579-0165175

29 Dowling S, Cox J and Cenedella RJ: Inhibition of fatty acid synthase by orlistat accelerates gastric tumor cell apoptosis in culture and increases survival rates in gastric tumor bearing mice in vivo. Lipids 44(6): 489-498, 2009. PMID: 19381703. DOI: 10.1007/s11745-009-3298-2

30 Carvalho MA, Zecchin KG, Seguin F, Bastos DC, Agostini M, Rangel ALCA, Veiga S, Raposo HF, Oliveira HCF, Loda M, Coletta RD and Graner E: Fatty acid synthase inhibition with Orlistat promotes apoptosis and reduces cell growth and lymph node metastasis in a mouse melanoma model. Int $\mathrm{J}$ Cancer 123(11): 2557-2565, 2008. PMID: 18770866. DOI: 10.1002/ ijc. 23835

31 Seguin F, Carvalho MA, Bastos DC, Agostini M, Zecchin KG, Alvarez-Flores MP, Chudzinski-Tavassi AM, Coletta RD and Graner E: The fatty acid synthase inhibitor orlistat reduces experimental metastases and angiogenesis in B16-F10 melanomas. Br J Cancer 107(6): 977-987, 2012. PMID: 22892389. DOI: $10.1038 / \mathrm{bjc} .2012 .355$

32 Garcia SB, da Costa Barros LT, Turatti A, Martinello F, Modiano P, Ribeiro-Silva A, de Oliveira Vespúcio, MV and Uyemura SA: The anti-obesity agent Orlistat is associated to increase in colonic preneoplastic markers in rats treated with a chemical carcinogen. Cancer Lett 240(2): 221-224, 2006. PMID: 16377080. DOI: 10.1016/j.canlet.2005.09.011
33 Cioccoloni G, Bonmassar L, Pagani E, Caporali S, Fuggetta MPIA, Bonmassar E, Atri SD and Aquino A: Influence of fatty acid synthase inhibitor orlistat on the DNA repair enzyme $\mathrm{O}^{6}$ methylguanine-DNA methyltransferase in human normal or malignant cells in vitro. Int J Oncol 47: 764-772, 2015. PMID: 26035182. DOI: 10.3892/ijo.2015.3025

34 Hong JL, Meier CR, Sandler RS, Jick SS and Stürmer T: Risk of colorectal cancer after initiation of orlistat: matched cohort study. BMJ 347: f5039, 2013. PMID: 23982291. DOI: 10.1136/ BMJ.F5039

35 Schcolnik-Cabrera A, Chávez-Blanco A, Domínguez-Gómez G, Taja-Chayeb L, Morales-Barcenas R, Trejo-Becerril C, PerezCardenas E, Gonzalez-Fierro A and Dueñas-González A: Orlistat as a FASN inhibitor and multitargeted agent for cancer therapy. Expert Opin Investig Drugs 27(5): 475-489, 2018. PMID: 29723075. DOI: 10.1080/13543784.2018.1471132

36 Yang PY, Liu K, Ngai MH, Lear MJ, Wenk MR and Yao SQ: Activity-based proteome profiling of potential cellular targets of orlistat - an FDA-approved drug with anti-tumor activities. J Am Chem Soc 132(2): 656-666, 2010. PMID: 20028024. DOI: 10.1021/ja907716f

37 Sriburi R, Jackowski S, Mori K and Brewer JW: XBP1: a link between the unfolded protein response, lipid biosynthesis, and biogenesis of the endoplasmic reticulum. J Cell Biol 167(1): 2004. PMID: 15466483 . DOI: $10.1083 /$ jcb.200406136

38 Cirillo L, Gotta M and Meraldi P: The Elephant in the Room: the role of microtubules in cancer in cell division machinery and disease. Gotta M and Meraldi P (eds.). Switzerland, Springer Int Publ. AG, pp. 93-124, 2017. PMID: 28600784. DOI: 10.1007/ 978-3-319-57127-0_5

39 Heuer TS, Ventura R, Mordec K, Lai J, Fridlib M, Buckley D and Kemble G: FASN Inhibition and taxane treatment combine to enhance anti-tumor efficacy in diverse xenograft tumor models through disruption of tubulin palmitoylation and microtubule organization and FASN inhibition-mediated effects on oncogenic signal. EBioMedicine 16: 51-62, 2017. PMID: 28159572. DOI: 10.1016/j.ebiom.2016.12.012

40 Li J, Gu D, Lee SSY, Song B, Bandyopadhyay S, Chen S, Konieczny SF, Ratliff TL, Liu X, Xie J and Cheng JX: Abrogating cholesterol esterification suppresses growth and metastasis of pancreatic cancer. Oncogene 35(50): 6378-6388, 2016. PMID: 15613445. DOI: 10.1038/onc.2016.168 